Revista Iberoamericana. Vol. LXIII, Núms. 178-179, Enero-Junio 1997; 99-113

\title{
SOBRE INDIOS, AFROAMERICANOS Y LOS RACISMOS DE SARMIENTO
}

\author{
POR \\ ElizABETH GARRELS \\ Masachusetts Institute of Technology
}

\begin{abstract}
Allá por 1989 el Presidente de EE.UU., George Bush, jactándose de lo que a él le interesaba entender como la victoria definitiva del capitalismo, habló triunfalmente de "un nuevo orden del mundo". Sin embargo, este nuevo orden del mundo, que se reconoce neoliberal, busca su ideología en el pasado - en "el orden de cosas" señalado por Foucault como emergente en el siglo XVIII. En 1990, después de frustrarse sus aspiraciones a la presidencia del Perú, el paladín del neoliberalismo Mario Vargas Llosa publicó, en inglés y en una revista estadounidense para lectores educados, acomodados y centristas, un tratado en contra del multiculturalismo titulado "Questions of Conquest: What Columbus wrought, and what he did not". Aquí el famoso novelista peruano se sitúa a la derecha en un debate cultural de gran actualidad no sólo para América Latina sino para los Estados Unidos. Entre otras cosas, Vargas Llosa se identifica con lo que él llama la tragedia de Domingo F. Sarmiento. La historia del escritor argentino que llegó a ser presidente de su país lo afecta, singularmente, por su tristeza:
\end{abstract}

He was totally convinced that the only way in which Argentina could become modern was through westernization; that is, through the elimination of everything that was nonWestern. He considered the Indian tradition, which was still present in the countryside of Argentina, a major obstacle for the progress and modernization of the country. He gave the moral and intellectual arguments in favor of what proved to be the decimation of the native population. That tragic mistake still looms in the Argentine psyche (53).

Inmediatamente antes escribe lo siguiente:

If forced to choose between the preservation of Indian cultures and their complete assimilation, with great sadness I would choose modernization of the Indian population .... It is tragic to destroy what is still living, still a driving cultural possibility, even if it is archaic; but I am afraid we shall have to make a choice ... where there is such an economic and social gap [como en América Latina], modernization is possible only with the sacrifice of the Indian cultures (52-53).

Vargas Llosa admite que su propia decisión es también trágica pero que la tomaría porque la considera necesaria y por lo tanto acertada. En cambio, dice que la de Sarmiento 
fue, además de trágica, un error. Según el peruano, Sarmiento entendió la occidentalización del indio no sólo como el exterminio de su cultura sino como el exterminio del indio mismo. Vargas Llosa, quien supongo se indignaría de ser llamado "racista", salva el cuerpo del indio (pero no su cultura) porque cree que el indio puede ser "reeducado". La solución de Vargas Llosa es la asimilación, que él llama, igual que el mexicano Vasconcelos en los años veinte, "mestizaje".

Con Vargas Llosa estamos una vez más ante un núcleo de cuestiones que en el Occidente no encuentra todavía el modo de superarse: por ejemplo, ¿hay categorías de personas naturalmente inferiores y por lo tanto incapaces de comportarse como seres plenamente racionales? Convencido de que así era, Aristóteles justificó su doctrina de la esclavitud natural. Dos mil años después, desde la segunda mitad del siglo XIX hasta el día de hoy, la biología y la ciencia social deterministas son capaces de afirmar la inferioridad intelectual de ciertas razas con respecto a otras. Para una mentalidad de ingeniería social (y no importa el siglo), la solución indicada bien puede ser o alguna forma de tutelaje, que incluye la esclavitud, o el exterminio. Si se cree que no hay correlación entre raza e inteligencia, la cuestión puede replantearse más nítidamente en el terreno de la historia y la cultura: ¿las diferentes culturas pueden o deben coexistir pacíficamente, o la cohesión social requiere cierto grado de homogeneización, realizado gradual o inmediatamente? Poco más de un siglo de intensos cambios históricos y profundas revoluciones tecnológicas separan las carreras intelectuales de Sarmiento y Vargas Llosa, pero el razonamiento -el discurso de los dos-parece, en lo fundamental, idéntico.

Tanto el Sarmiento decimonónico como el Vargas Llosa contemporáneo confieren un valor providencial al monoculturalismo. En sus Viajes de 1849, Sarmiento escribía que todos los pueblos del mundo marchaban, aunque a diferentes velocidades, a convertirse en "un mismo pueblo ... con el mismo vestido, las mismas ideas, las mismas leyes y constituciones, los mismos libros, los mismos objetos de arte" (139). ${ }^{1}$ Idem Vargas Llosa en 1990. Sin embargo, el escritor peruano sugiere que Sarmiento, a diferencia de él, creía en la equivalencia de raza y cultura; o sea, que había razas incapaces de ser reeducadas y así asimiladas a las prácticas culturales de otras razas. En su comentario sobre una obra del chileno Lastarria en 1844, Sarmiento parecía confirmar tal sugerencia. Allí justificaba la Conquista española del Nuevo Mundo y el exterminio de los indios por los españoles. Gracias a esta violencia exterminadora, escribía, "la América, en lugar de permanecer abandonada a los salvajes, incapaces de progreso, está ocupada hoi por la raza caucásica, la más perfecta, la más intelijente, la más bella i la más progresiva de las que pueblan la

\footnotetext{
${ }^{1}$ Comparése con lasiguiente descripción (que aparece en un reciente número de la revista estadounidense The Nation) de los efectos de la globalización hoy en día: "The guiding principles of the new economic structures assume that all countries - even those whose cultures have been so diverse as, say, Indonesia, Japan, Kenya, Sweden and Brazil- must row their (rising) boats in unison. The net result [...] is a global monoculture - the homogenization of culture, lifestyle and level of technological immersion, with the corresponding dismantlement of local traditions and self-sufficient economies. Soon, everyplace will look and feel like everyplace else, with the same restaurants and hotels, the same clothes, the same malls and superstores, the same TV, the same streets choked with cars and the same universal materialistic values. There'll scarcely be a reason ever to leave home" (Mander 10).
} 
tierra" (214). Un año después, en el Facundo, volvía a insistir en que el indio americano era incapaz, aun con la coerción, de superar su indolencia congénita y adquirir una ética de trabajo.

Al final de su vida, la perspectiva de Sarmiento sobre el indio seguía básicamente sin alterar, sólo que ahora estaba aderezada con la dudosa autoridad del cientificismo determinista finisecular. En su otoñal Conflicto y armonía de las razas en América, Sarmiento generalizaba del siguiente modo sobre negros e indios:

Los negros son capaces de entusiasmo y sensibles a muchos buenos estímulos. El indígena, salvo antagonismos de raza, es menos susceptible (115).

Aquí parece que el viejo defensor de la supremacía blanca valoraba al negro por encima del indio, opinión nada aislada entre los eurocéntricos del siglo pasado. A manera de ejemplo, en la Filosofia de la historia basada en sus conferencias de 1830-31, Hegel decía que "the negroes are far more susceptible of European culture than Indians" (82), y el Sarmiento de 1841 había escrito que el "negro de Africa central no es en todo respecto tan salvaje como el indio de América" (199).

Sin embargo, cuando el septuagenario habla de los indios que habitan el territorio argentino en 1883 (fecha de publicación del primer volumen de Conflicto), distingue entre tres tipos o ramos de indígenas. Estos son la "raza" quichua o peruana, la guaraní o misionera, y la pampa o araucana $(I, 35)$. Sarmiento jerarquiza a estos tres grupos, utilizando como criterio el grado de docilidad inherente que él asigna a cada uno. Los quichuas (quechuas) son los que en el siglo XVI se encontraban en las zonas de fundación de Santiago del Estero, Tucumán, Córdoba, Salta, y Jujuy, o sea el territorio que en el siglo XIX corresponde al noroeste de la República $(I, 39)$. Según el autor, estos indios son los más dóciles y obedientes de los tres grupos: "[durante] tres siglos han obedecido al primero que se propuso mandarlos" (I, 39). Esta docilidad, que para Sarmiento (igual que para Vargas Llosa en 1990) se traduce en la incapacidad de pensar de manera independiente, vuelve al quichua incapaz de funcionar como ciudadano de una república. El quichua es:

... un siervo tan manso, tan dócil, tan sumiso, que no puede hacerse de él un ciudadano, sirviendo por el contrario, sin necesidad de esclavizarlo, para ayudar a despojar sus libertades a los blancos, sin odio y simplemente por pura obediencia (II, 115).

Los guaraníes, cuyos descendientes se encuentran, en 1883, en Corrientes, Entre Ríos, Uruguay y el Brasil, son también sumisos pero lo son menos que los quichuas $(\mathrm{I}, 45)$. Los araucanos son el tercer grupo e incluyen a los pampeanos, ranqueles, etc., del sur. Son los que durante los primeros sesenta años de la República constituyen el muy mentado "problema de frontera". Con sus guerras intermitentes y sus malones a las poblaciones cristianas de habla española, los arauco-pampeanos, hasta la Campaña del Desierto de 1879 (o sea, la "solución definitiva" optada por el gobierno argentino) eran "el terror de nuestras fronteras, hasta que una vez por todas se resolvieron nuestros generales y gobernantes a destruirlos" (I, 53). Según el ex-presidente de la República, los arauco-pampeanos eran, en comparación a los otros dos grupos, "más indómitos, lo que quiere decir: animales más reacios, menos aptos para la civilización y asimilación europeas" (I, 54). 
Con esta jerarquía se descubre que hay, grosso modo, dos tipos de indios. Uno resulta ciegamente sumiso y obediente, y puede ser domado, domesticado, asimilado y civilizado en la medida en que sea apto para trabajar como siervo o peón y para pelear y morir como soldado en los ejércitos encabezados por blancos. Este es asimilable en posición de inferioridad a la civilización blanca, pero es congénitamente incapaz de habilitarse para la plena ciudadanía en una democracia republicana. El otro tipo, el araucano - el de los malones y, durante unos treinta años antes de la Campaña exterminadora de 1879, el de las temibles confederaciones guerreras - no es ni asimilable, en estado domesticado de inferioridad servil, ni apto para la ciudadanía. ${ }^{2}$ Para el viejo Sarmiento, quien escribe en Buenos Aires para un público argentino, no todo indio merece ser colgado como lo había sugerido en 1844 el joven exilado viviendo en Chile y comentando un texto de Lastarria para un público chileno. ${ }^{3}$ Según nos cuenta el viejo en Conflictos, los chilenos construyen su patriotismo en base a una idealización de los araucanos literarios cantados por el poeta Ercilla; por lo tanto, el argentino polemizando en Chile sobre indios (tanto en la reseña del texto de Lastarria, en 1844, como en su Facundo, en 1845) parece reducir al indio tanto de Chile como de la Argentina al araucano indómito. ${ }^{4}$

En Conflicto, Sarmiento complica un poco su cuadro de los indios argentinos. Los quichuas del noroeste y los guaraníes del noreste son indios sumisos y obedientes (léase "asimilados ya" o "capaces de asimilación"), aunque son menos fieles que los negros. Los huarpes de San Juan, el lugar de origen del propio Sarmiento, aunque no son quichuas (según él), también pertenecen al grupo de los sumisos y asimilados. Recordemos que Sarmiento había hablado con cierta benevolencia paternalista de los huarpes en sus Recuerdos de 1850, donde elogiaba (como lo había hecho antes en el Facundo) al rastreador huarpe Calíbar.

Cuando Sarmiento habla de los negros "puros"(léase "africanos"), resalta las cualidades de la obediencia y la fidelidad. Como los indios sometidos y serviles, los negros son obedientes, pero a diferencia de ellos, se distinguen por su fidelidad al amo:

Los negros tienen cierta nobleza aun en la esclavitud, siendo calidad esencial de su caracter, la fidelidad (Conflictos, II, 185). ${ }^{5}$

\footnotetext{
${ }^{2}$ Reciententemente, la importante investigación de Kristine L. Jones nos ha facilitado entender mejor las complejas relaciones en el siglo XIX entre la sociedad blanca/mestiza de la Argentina y la araucopampeana, incluyendo el grado notable de transculturación y mutua dependencia económica que se alcanzó entre las dos. Se recomiendan sus dos ensayos "Indian-Creole Negotiations in the Southern Frontier" y "Civilization and Barbarism and Sarmiento's Indian Policy".

${ }^{3}$ En su reseña, ya comentada, de "Investigaciones sobre el sistema colonial de los españoles", Sarmiento había escrito: "para nosotros Colocolo, Lautaro i Caupolicán, no obstante los ropajes civilizados i nobles de que los revistiera Ercilla, no son más que unos indios asquerosos, a quienes habríamos hecho colgar i mandaríamos colgar ahora, si respareciesen en una guerra de los araucanos con Chile[...]" (214).

${ }^{4}$ En Conflicto (I, 56), se lee: "La historia de Chile está calcada sobre la 'Araucana', y los chilenos, que debían reputarse vencidos con los españoles, se revisten de las glorias de los araucanos a fuer de chilenos estos, y dan a sus valientes tercios el nombre de Carampangui y a sus naves el de Lautaro, Colocolo, Tucapel, etc". En el Facundo, se lee: "Las razas americanas se muestran incapaces, aun por medio de la compulsion, para dedicarse a un trabajo duro y seguido" (28).

${ }^{5}$ En el Facundo había escrito: "Los africanos son conocidos por todos los viajeros como una raza guerrera, llena de imaginación y de fuego, y aunque feroces cuando están exitados, dóciles, fieles y adictos al amo o al que los ocupa" (217).
} 
Sarmiento escribe en el segundo volumen (póstumo) de Conflictos que en el siglo XVIII había pocos indios de servicio en la ciudad de Buenos Aires porque no merecían la confianza de los blancos. En cambio, durante la misma época había ocho negros por cada blanco (II, 185).

Aunque los "puros" negros resultan superiores a los "puros" indios quichuas o guaraníes por ser fieles, tampoco sirven, según dice Sarmiento en varios escritos de distintas épocas y diferentes contextos, para ciudadanos republicanos. En el Facundo, de 1845, escribe que los negros "[encuentran] su patria y su manera de gobernar, en el amo a quien [sirven]" (218). ${ }^{6}$ En Viajes, de 1849, escribe que "el negro [es] incapaz de elevarse a las altas regiones de la civilización" (62). Y en 1869, siendo Presidente de la Repúblicay escribiendo desde Buenos Aires, describe al negro como "niño que canta, ríe, baila y obedece. Dios lo dejó así, a medio crecimiento" (302).

Durante su segunda estadía en Estados Unidos (mayo de 1865 a 1868), en que le tocó conocer el período más militante de la llamada Reconstrucción del sur (inmediatamente después del término de la Guerra Civil y del asesinato de Lincoln), Sarmiento elogiaba las labores de la Asociación de Ayuda para los Libertos. Este era un grupo filantrópico de blancos en el norte de USA que se dedicaba a establecer escuelas para libertos en el sur con el fin de "instruirlos en el deber, y hacerlos útiles" y, por si acaso el estado decidía concederles a los hombres negros el sufragio, con el fin de que tuvieran "ciencia y conciencia de sus actos" ("Educación" 234). En Washington, D.C., en Nueva York, y en Boston, Cambridge y Concord, el Ministro Plenipotenciario en Estados Unidos-nombrado para representar el nuevo gobierno nacional de la Argentina- entraba en contacto no sólo con Eduardo F. Davison, cónsul argentino en Nueva York y miembro de la Comisión Ejecutiva de la Asociación de Ayuda para los Libertos; entraba en contacto además con toda la oficialidad norteamericana que recién salía triunfante de la guerra. Aprovechando su amistad ya establecida con Mary Mann (viuda de Horace Mann), se enorgullecía de tratar con intelectuales estadounidenses de la talla del abolicionista y filósofo transcendentalista Ralph Waldo Emerson. Lo escrito por Sarmiento en esta época-que incluye su Introducción a una Vida de Abraham Lincoln - tendría que estar, juzgado desde un punto de vista contemporáneo, entre lo menos teñido de racismo que Sarmiento produjera sobre el negro durante su larga carrera de escritor. ${ }^{7}$ En estos artículos y cartas desaparece la distinción que el autor manejaba en el Brasil en 1846 (aunque no en Chile apenas un año antes): la del mulato como un ser diferente de y superior al negro "puro". En lo escrito desde USA durante la Reconstrucción, parece que no le importa a Sarmiento la cuestión de la pureza de la sangre africana.

En los años sesenta, Sarmiento calcula que EE.UU. tiene cuatro millones de negros. Para 1883, le calcula un millón más:

\footnotetext{
${ }^{6}$ Sarmiento dice esto al hablar de la adhesión de los negros de Buenos Aires a Rosas. Sobre las relaciones establecidas entre la población negra del puerto y el dictador Rosas, ver Lynch y Kelly.

${ }^{7}$ Por esos mismos años Sarmiento calificaría a Lincoln como "la figura más noble de los tiempos modernos" ("Base" 236).
} 
... la independencia de la raza blanca eliminó la raza negra en toda la extensión del continente, mientras sólo queda libre en los Estados Unidos, en número de cinco millones, después de una guerra social; en La Habana queda esclava, después de un supremo y malogrado esfuerzo de la raza blanca criolla para emanciparse [Se refiere a la Guerra de Diez Años, de 1868 a 1878], y esclavos quedan en el Brasil los negros que hoy existen, sin transmisión de la servidumbre a sus hijos (I, 64).

Escribe que en la Argentina, "como raza, como elemento social, no son ya sino un accidente pasajero, habiendo desaparecido del todo en las provincias" (I, 68), y que, "De Buenos Aires en veinte años más, será preciso ir al Brasil para verlos en toda la pureza de su raza" (I, 65). Sea cierta o no la percepción de que los afroargentinos desaparecieron como grupo en menos de cincuenta años durante el siglo pasado, para Sarmiento esta percepción logra el mismo fin que la Campaña del Desierto de 1879 logró respecto al indio: tanto el consenso sobre un modo de ver como la implacable campaña militar borran del suelo argentino a dos razas de color que en su "pureza" son tenidas por Sarmiento como biológicamente inferiores al blanco y por lo tanto como impedimentos al progreso de la nación. ${ }^{8}$ Este prejuicio, que Sarmiento articula sin pelos en la lengua en la década de los ' 80 , puede dar la clave para entender aquella declaración suya tan elíptica del penúltimo capítulo del Facundo de 1845, donde escribía que, "Felizmente, las continuas guerras han exterminado ya la parte masculina de esta población [negra]" (218). "Felizmente" para la civilización europea, el progreso y la democracia republicana porque de las tres razas de que se hizo el tipo popular argentino de principios de siglo, dos de ellas - la negra y la india- son, en su "pureza", radicalmente inferiores a la raza blanca "pura". En tal jerarquía supuestamente natural y determínante, el tipo intermedio entre el color y la blancura, o sea, el mestizo y el mulato, es sólo redimible como ser pensante en proporción a su cantidad de sangre blanca.

Detengámonos ahora en este tipo intermedio, es decir, en la cuestión de la mezcla racial. Tanto Sarmiento, al describir a la Argentina en los años cuarenta, como su modelo intelectual el francés Alexis de Tocqueville, al describir a los Estados Unidos en el primer lustro de los treinta, manejaban el patrón del trinomio para describir la composición racial/ cultural de la realidad americana sometida a su examen. "Las tres razas que habitan el territorio de los Estados Unidos," del Cap. XVIII, Tomo I, de Tocqueville serían, en el primer capítulo del Facundo, las "tres familias" que, fundiéndose, han producido "un todo homogéneo" en la Argentina. Para los dos autores, la raza blanca era la históricamente triunfante, aunque Sarmiento no expresaba nada del respeto, simpatía, ni sentimiento por las injusticias cometidas contra las razas perdedoras (especialmente la indígena) que expresaba Tocqueville. ' Tocqueville escribe que o la mezcla de razas o la separación total serían la únicas soluciones pacíficas a la cuestión racial en Estados Unidos:

I have previously observed that the mixed race is the true bond of union between the Europeans and the Indians; just so, the mulattoes are the true means of transition between

\footnotetext{
${ }^{8} \mathrm{El}$ asunto de la desaparición de los negros en Buenos Aires ha sido estudiado en Andrews.

${ }^{9}$ Sobre la activa simpatía que Tocqueville expresa por los indios americanos, véase Democracy in America 344, 359, 363.
} 
the white and the Negro; so that wherever mulattoes abound, the intermixture of the two races is not impossible $(\mathrm{I}, 389)$.

Dice que en algunas partes de las Américas se ha dado tanta mezcla entre blancos y negros que es difícil encontrar un hombre "who is entirely black or entirely white". En estos casos, se ha creado "una tercera raza" ("a third race, which is connected with both without being identical with either" [389]). Esta posibilidad, sin embargo, no se dará en Estados Unidos porque, entre otras cosas, "Of all Europeans, the English are those who have mixed least ..." (389).

Es un lugar común, incluso entre los que rechazan los varios mitos de la democracia racial latinoamericana, el que exista una diferencia fundamental entre los respectivos racismos de Estados Unidos y América Latina. En Estados Unidos, una gota de sangre africana tradicionalmente ha condenado a uno a la inferioridad social (o sea, que una fracción mínima de sangre africana ha tenido un mayor poder social negativo que el efecto positivo que supuestamente podría tener un porcentaje mayoritario de sangre caucásica). Al contrario, en las zonas latinoamericanas de mayor presencia africana, suele funcionar la noción opuesta del blanqueamiento (o sea, que cualquier porcentaje de sangre caucásica, por pequeña que sea, corrige de manera proporcional los defectos debidos a la sangre negra). Pidiendo prestado el término de Tocqueville, se podría decir que, grosso modo, en Estados Unidos, no se ha admitido entre los que se consideran blancos la posibilidad de una tercera raza intermedia. ${ }^{10}$

Por lo menos en la década de los cuarenta, la mentalidad racista de Sarmiento parecería ser consistente con el criterio de blanqueamiento como mejoramiento racial. En varios textos de esta década, encontramos un elogio del mulato precisamente porque el mulato, según Sarmiento, es capaz de asimilarse a la cultura eurocéntrica que él considera la más perfecta de todas. Sarmiento jamás concibe que las culturas africanas o afroamericanas pudieran tener una validez social igual a la de la europea o la europea-americana; siempre las califica de salvajes, bárbaras o primitivas.

Como ya se dijo, Sarmiento cree que tanto el indio "puro" como el negro "puro" son biológicamente inferiores e incapaces del progreso. Sin embargo, si se estudian sus textos aislados, es posible confundirse con respecto a lo que Sarmiento opina sobre el negro. Quiero dejar constancia de que de aquí en adelante en mi discusión, usaré la terminología de Tocqueville que reconoce en el negro y el mulato dos categorías diferentes, y no la corriente en Estados Unidos que de hecho sólo admite la existencia del negro y no la del mulato. (Sostengo que los términos "raza pura", "negro puro", "blanco puro", etc., sólo tienen una realidad ideológica, y no representan ninguna realidad biológica objetiva. Es por esta razón que he venido colocando estos términos entre comillas).

En dos textos de 1845, sus Apuntes biográficos sobre Aldao, de febrero, y su Facundo, cuya publicación periódica comienza en mayo, Sarmiento no distingue claramente entre el

\footnotetext{
${ }^{10}$ En zonas que culturalmente pertenecen al mundo plantócrata caribeño al igual que a Estados Unidos, como Luisiana y en particular la ciudad de Nueva Orleans, la valoración social de las distintas graduaciones de color que en los siglos XVIII y XIX imperaba en el Caribe hispano tenía una vigencia relativa.
} 
negro y el mulato. De hecho, toda distinción desaparece cuando elogia al Coronel Barcala, un liberto criollo nacido en Mendoza, quien se da a conocer en la guerra contra el Brasil de 1826-28, después sirve bajo el general unitario José María Paz cuando éste controla la ciudad de Córdoba entre 1829 y 1831, luego es tomado prisionero y sirve bajo el caudillo federal Juan Facundo Quiroga, y termina fusilado por el federal Aldao. En el primer texto, Sarmiento lo describe como "un negrito criollo esclavo, que desde temprano había manifestado el talento y despejo que no es raro ver en los descendientes de raza africana"; habla "[d]el genio de que la naturaleza le había dotado"; dice que es "una de las figuras más distinguidas de la revolución argentina, y una de las reputaciones más intachables" y que "era un hombre eminentemente civilizado en sus maneras, gustos e ideas"; lo llama el "virtuoso negro" y "el caballero negro" (Aldao 22-24). Es siempre el "negro" o el "liberto", pero nunca el "mulato". Lo mismo ocurre en las frases, también elogiosas, que Sarmiento le dedica a Barcala en el Facundo; allí es "el ilustre negro", "el liberto consagrado, durante tantos años, a mostrar a los artesanos [de Córdoba] el buen camino, y a hacerles amar una revolución que no distinguía ni color ni clase para condecorar el mérito" (143)."

Si uno no sabe que Barcala era mulato, sólo lo averigua leyendo la carta que Sarmiento fechó el 20 de febrero de 1846, en Río de Janeiro, y que luego publicó en sus Viajes de 1849. Allí Sarmiento escribe:

El mulato se levanta ya en el Brasil amenazando vengar bien pronto las injurias hechas a su tostada madre. Raza viril que conserva la sangre ardiente del africano, templada para bullir bajo los rayos verticales del sol, al mismo tiempo que la organización de su cráneo lo liga a la familia europea. Dumas, Heredia, Pétion, Barcala, aquellos nobles mulatos, viven aquí en todos cuantos hombres notables brillan por las artes, la música, la poesía y las ciencias médicas....

Me detengo ... sobre las brillantes cualidades morales de esta raza intermediaria entre el blanco que se enerva en los climas ecuatoriales y el negro incapaz de elevarse a las altas regiones de la civilización. Otra vez había notado la predisposición constante del mulato a ennoblecerse, y su sentimiento exquisito del arte, que lo hace institivamente músico. Viénele la primera cualidad de haber ensanchado su frente, y la segunda de la sangre africana que calienta su nuevo y mas idóneo cerebro (62-63).

Aquí se dice a las claras que "el negro [es] incapaz de elevarse a las altas regiones de la civilización". Esta supuesta incapacidad la comparte con el indio. Pero aquí también se recurre al concepto de una tercera raza o de una raza intermediaria.

La ausencia del concepto de mulato explícitamente delineado en el Facundo se presta a varias confusiones. Sin este concepto de una raza intermediaria, el discurso sobre el negro en el libro más famoso de Sarmiento resulta plenamente contradictorio. La primera vez que se refiere al negro en el Facundo, al hablar de "las tres familias" que componen la población del país, se describe a la "raza negra" como "casi extinta ya —excepto en Buenos Aires".

\footnotetext{
"Según Emiliano Endrek, estos artesanos en Córdoba serían en su mayoría mestizos "secundarios" o sea, mezclas de español, indio y negro (22). Durante la Colonia, el contingente indígena era bastante reducido en el área de Córdoba, que en cambio contaba con un elevado número de esclavos. Las castas “constituían la mayoría del artesanado colonial" (26).
} 
Se señala que el negro sin embargo ha contribuido a formar la población argentina con sus "zambos y mulatos, habitantes de las ciudades, eslabón que liga al hombre civilizado con el palurdo, raza inclinada a la civilización, dotada de talento y de los más bellos instintos de progreso" (28).

La imprecisión de la última referencia fácilmente confunde al lector. Al principio de la oración, se habla de "la raza negra", aclarando que ésta sólo perdura de manera significativa en Buenos Aires. Sin embargo, en las ciudades (¿Serán las otras trece "ciudades capitales de provincia" de las que se habla a continuación?) habitan zambos y mulatos, "eslabón que liga", o sea, raza intermediaria. ¿Es ésta la raza inclinada a la civilización, o es la negra? Si cotejamos diferentes textos escritos por Sarmiento en estos años, creo que hay que concluir que se refiere a los mulatos. Sin embargo, esta oración tan elíptica del primer capítulo del Facundo hace posible pensar que se trata también de negros y zambos, estos últimos el producto de enlaces entre negros e indios, dos razas condenadas a la inferioridad biológica por el racismo del autor. Más adelante en el Facundo, cuando Sarmiento habla del noble Barcala, lo identifica como "negro". Al final del libro, en la Parte II, Cap. 10, cuando habla de cómo el dictador Rosas ha perdido la adhesión de la campaña (Léase "el gaucho o el pueblo mestizo"), dice que ha buscado el apoyo de "dos pueblos distintos, de razas diversas" (217). Se está refiriendo al indio y al negro como razas "puras"; el uno es el arauco-pampeano no asimilado, y el otro es el negro africano, quien por no ser criollo tiene menos probabilidad de ser mulato. En el caso de los negros, se trata de la "multitud de negros [africanos], de los millares quitados por los corsarios [de los buques negreros brasileños], durante la guerra del Brasil" (217). "Felizmente", escribe el autor, "las continuas guerras han exterminado ya la parte masculina de esta población, que encontraba su patria y su manera de gobernar, en el amo a quien servía”. Siendo aquí Rosas el amo, la virtud atávica de la fidelidad del negro se había convertido en una amenaza concreta a la política de Sarmiento. También, al exterminarse la parte masculina de la población africana, quedaban supuestamente disponibles las mujeres del grupo. Estas, pues, serían las "tostadas madres" de futuras generaciones blanqueadas y mejoradas de mulatos, ya que Sarmiento y tantos otros situaban, de manera explícita, el origen de la mezcla racial en la mujer de color $\mathrm{y}$ en el hombre blanco.

En el Sarmiento de los años cuarenta, el mulato y el negro - aunque no siempre se articule con claridad - representan dos seres racial y culturalmente distintos. El negro, como el indio arauco-pampeano sin mezcla, son seres incapaces de evolucionar para "occidentalizarse" o "modernizarse" (los términos de Vargas Llosa) o para convertirse en responsables ciudadanos republicanos (el ideal tal como lo articularía Sarmiento). En cambio, el mulato funciona como un sujeto capaz de distinguirse por el mérito, o sea, un tipo social que puede aprovechar las oportunidades abiertas por la revolución democrática moderna y por el derrocamiento de las jerarquías del antiguo régimen. Para Sarmiento, el mulato es el tipo Benjamín Franklin proyectado al discurso de la raza. De hecho, los dos panegíricos dedicados a Barcala en 1845 son, entre otras cosas, defensas del mérito individual como criterio social. Este es el mismo criterio mediante el cual el Sarmiento de 1843, en su primera autobiografía (Mi defensa), insiste en valorarse a sí mismo. El es el oscuro exilado, el provinciano autodidacta y el miembro de una familia "decente" venida a menos, que aboga apasionadamente por el criterio del mérito individual del "self-made 
de este criterio social democrático que Sarmiento puede, implícitamente, identificarse con el mulato Barcala o con el protagonista del drama romántico del famoso dramaturgo y novelista francés, Alejandro Dumas (padre), mulato él mismo por ser nieto del enlace ilegítimo entre el Marqués de Pailleterie y la negra Marie Cessette Dumas en la colonia francesa de Saint Dominque (luego Haití). En una reseña del drama "El mulato" de Dumas, fechada el 15 de julio de 1842, es obvio que Sarmiento generaliza el dilema del héroe mulato a todo "joven honrado, de talento i de una mediana fortuna" que quisiera ganar la mano de una hija "de familia de cierto calibre i alcurnia" sin que el padre de la muchacha pregunte primero "quién fué su padre, i de qué familia desciende" (280). En el mismo mes de julio, en una de sus contribuciones a la famosa polémica sobre el romanticismo vs. el clasicismo, Sarmiento se suscribirá a los principios de "la nueva escuela socialista" (el sansimonismo de Pierre Leroux y la Revue Encyclopedique), afirmando que ya es hora de "avergonzarse de que el plebeyo, el mulato, con talento, con virtudes, sea despreciado i mantenido en una inferioridad inmerecida" ("Continúa el examen" 304).

En la defensa del mulato que se incluye en el capítulo "Río de Janeiro" de Viajes, Sarmiento discute la palabra "mulato" usada como insulto contra un individuo supuestamente blanco. Ya en su reseña de la obra de Dumas, Sarmiento había disertado sobre la etimología insultante de esta palabra:

... mulato trae su orijen de mula, mezcla de dos razas distintas que producen entre el caballo i el burro la mula, i entre el hombre blanco i la mujer negra el mulato. Ya verán nuestros mulatos todo el honor que les han hecho los caballeros que inventaron la palabra (280).

En Río de Janeiro, centro político y cultural de la monarquía brasileña y ciudad que en 1849 tenía 78.000 esclavos (Klein 129), Sarmiento escribía:

La raza pura portuguesa cae visiblemente en la decrepitud y en la inanición, y en las cámaras y en la prensa diaria, más fecunda aquí en injurias que entre nosotros, todo se dicen los contendientes, hasta sodomitas, menos mulatos; porque cada uno se siente implicado en el reproche, en sus hijos, en sus deudos o en sí mismo. Hay una ley que prohíbe el uso de este epíteto, medida segura para pesar la gravedad del mal (62).

En cambio, en su nativo San Juan de la Frontera, de la Argentina, la palabra "mulato" circulaba todavía como "calumnia" utilizada por familias "españolas" no fundadoras para desprestigiar a los descendientes de uno de los primeros pobladores de la ciudad, el "hijodalgo Mallea," quien en el siglo XVI se había casado con "la hija del cacique de Angaco"(Sarmiento, Recuerdos 11). En su segunda autobiografia, Recuerdos de provincia, de 1850, Sarmiento escribía:

¡Decíanles mulatos! y yo los he alcanzado luchando todavía contra esta calumnia que se transmitió de padres a hijos. Mi madre ... me cuenta que don Luciano Mallea ... era muy conocedor en genealogías, y sostenía que eran ellos mestizos de pura y noble sangre (21).

En esta autobiografía tan saturada de añejos prejuicios coloniales, no se encuentra la defensa del mulato sino la aceptación tácita de que llamar "mulata" a la "gente decente" es 
calumnia. ${ }^{12}$ (Hasta se encuentra la repetición "inocente" del oximoron "mestizos de pura sangre").

Además de la profunda historicidad que se hace ver en la yuxtaposición de estos dos comentarios de Sarmiento sobre el uso insultante de la palabra "mulato", me interesa señalar aquí su observación sobre el silencio como censura. Lo que Sarmiento dice sobre la supresión de "mulato" como insulto en Río de Janeiro revela una conciencia, diríamos "protopsicoanalítica," de que las palabras prohibidas a menudo se refieren a lo más importante y a la vez más amenazante para uno. Esta observación de Sarmiento permite hablar de un silencio en la estructura de su libro Viajes, silencio que sospecho dice mucho sobre la complejidad de su actitud hacia el afroamericano. Me refiero a su silencio sobre su viaje a Cuba a fines de 1847.

El traductor de la sección sobre Estados Unidos, Michael Rockland, ha criticado a Sarmiento por el "virtual" silencio en Viajes sobre la esclavitud en ese país (45). Tal silencio, sin embargo, no es tan extremo como lo sugiere Rockland, aunque sí concedo que el espacio que Sarmiento dedica a la esclavitud en USA es comparativamente reducido. Yo insistiría, más bien, en la importancia de que el autor coloque su comentario más extenso sobre el tema justamente al final del texto, dándole así la categoría privilegiada de "las últimas palabras". También llamaría la atención sobre el tono apocalíptico de estas palabras, el cual les da una importancia que contrarresta su brevedad.

Además de hablar de la esclavitud en USA, Viajes habla de la esclavitud en el Brasil, aunque aquí también es cierto que le interesa más hablar del paisaje tropical y del diseño urbano de Río de Janeiro. Para 1850, año en que el Brasil terminaría su trata negrera, el país habría recibido más esclavos africanos que cualquier otro país de las Américas (Skidmore 8-9). Sin embargo, debido a una reproducción natural menos exitosa de la población esclava en el Brasil que en USA, el Brasil de mediados del siglo XIX era, con unos 1.7 millones al final de los años cincuenta, el estado esclavista más grande de Latinoamérica pero el segundo del hemisferio (Klein 157). Estados Unidos era el más grande, con 4 millones de esclavos al comenzar su guerra civil en 1861. La estadía de Sarmiento en el Brasil corresponde al comienzo de su viaje. Después de zarpar de Chile el 28 de octubre de 1845, Sarmiento visita los puertos sudamericanos de Montevideo y Río antes de cruzar el Atlántico para Europa.

Los dos últimos capítulos de Viajes están dedicados a Estados Unidos, pero Estados Unidos no es el último país que Sarmiento visita antes de regresar a Chile. Después de abandonar USA por el puerto de Nueva Orleans, ciudad esclavista que le provoca al viajero

\footnotetext{
${ }^{12}$ Recuerdos es también el libro en que el liberal Sarmiento, que generalmente hace suya la retórica del abolicionismo gradualista, escribe que en la niñez colonial de su madre, "la esclavitud no envilecía las buenas cualidades del fiel negro" (31-32). Para una discusión ya clásica sobre la mentalidad aristocrática/colonial de Recuerdos, ver Halperín Donghi. Sobre la escala de racismos en la Argentina colonial del siglo XVIII, que discrepaba directamente con la defensa ocasional por parte de Sarmiento, ya en época poscolonial, del mulato, ver Endrek. Endrek cita a Juan Solórzano y Pereyra, quien escribía en su Politica Indiana de 1776 que el tipo de mestizo peor considerado en la colonia era el hijo de negra y blanco o viceversa "por tenerse esta mezcla por más fea y extraordinaria, y dar a entender con tal nombre, que le comparan a la naturaleza del mulo[...]" (6).
} 
su profecía tan alarmada sobre el futuro del país, Sarmiento viaja a Cuba. ${ }^{13}$ Desembarca en La Habana el 15 de noviembre. Si damos fe a su Diario de Gastos, parece quedarse unas tres semanas en el centro administrativo de Cuba, la más rica de las pocas colonias que le quedaban a la antigua Madre patria España. Luego Sarmiento pasa a Santiago de Cuba, puerto de una de las cuatro provincias cubanas de mayor producción azucarera y por lo tanto de mayor concentración esclava rural (Knight 41). ${ }^{14}$ Es probable que abandone a Cuba por este puerto al suroeste de la isla. Después pasará a Panamá, para cruzar el istmo a caballo y luego descender por el Pacífico hasta Lima, Arica y Valparaíso, a donde arriba en octubre de 1848. Comenzando con la omisión de Cuba, el último trecho del largo viaje de Sarmiento queda sumergido en un silencio textual absoluto.

El libro Viajes termina con el Sur esclavista de USA y con una subida retórica religiosa y moralista muy característica del abolicionismo internacional; ésta anuncia un posible castigo divino a la sociedad estadounidense por el pecado de practicar la esclavitud. El viajero, sin embargo, sigue su viaje, y de USA pasa directamente al tercer estado esclavista más grande del hemisferio y al segundo en tamaño en Latinoamérica. La Cuba española que visita y que no comenta en absoluto es la Cuba de finales de 1847. Su industria azucarera está a punto de cumplir una década clave - una década en que se ha visto una verdadera revolución tecnológica debida al comienzo de operaciones de los ferrocarriles en 1838 y al incremento del uso de la energía de vapor en los ingenios. ${ }^{15}$ A partir de 1838 , la industria azucarera monopoliza la economía de la isla; la industria se reorganiza profundamente y casi todas las plantaciones más grandes y eficientes de todo el siglo XIX o se fundan o se reorganizan en esta década que va de 1838 a 1848 (Knight 39).

Sarmiento llega, pues, en pleno apogeo del azúcar en Cuba. Y hasta cumplida la Guerra de Diez Años en 1878, el azúcar en Cuba significará la esclavitud negra. Sarmiento llega en un momento en que la cuestión de la esclavitud ocupa un lugar singularmente central y obsesivo tanto en la política como en el imaginario de los blancos de la isla. Recién se había cerrado una etapa de intensos disturbios raciales para Cuba (1842-43), la cual culminó en la denunciada Conspiración de la Escalera en 1844. Tal conspiración, que nunca logró materializarse en rebelión, de todos modos dio al gobierno una excusa para intensificar la persecución de la gente libre de color y la opresión de los esclavos (Klein 212-13). Para el año 1845 las rebeliones esclavistas y las conspiraciones ya estaban reprimidas, pero ahora los plantócratas encontraban un nuevo motivo de pánico en la Ley de la Abolición y Represión de la Trata de Esclavos promulgada el mismo año. Aunque España había encontrado la manera de no respetar un tratado con objetivos afines en 1835, decidió esta vez cumplir -más o menos- su palabra. Entre 1845 y 1850, cuando el gobierno dejó de nuevo de interferir en la trata, el número de africanos importados a Cuba desde Africa bajó dramáticamente. La ley de 1845 provocó protestas vigorosas por parte de los plantócratas, y hasta hizo que algunos de ellos vendieran sus tierras y esclavos, creyendo que la abolición de la esclavitud era inminente (Knight 139-42).

\footnotetext{
${ }^{13}$ Esta profecía hace eco de, y hasta imita retóricamente, la profecía que su obvio modelo Tocqueville coloca hacia el final del primer volumen de Democracia en América, de 1835.

${ }^{14}$ Aquí Knight habla específicamente de 1871.

${ }^{15}$ El ferrocarril de 1838 es el primero que se construye en toda la América Latina. Según Knight (39), para $1846,19.8 \%$ de los ingenios serán activados por el vapor, un salto desde $2.5 \%$ en 1827 .
} 
Si el viaje a Estados Unidos significó para Sarmiento un viaje al futuro, ${ }^{16}$ el viaje a la colonia española de Cuba debe haber significado algo como un viaje al pasado. Pero era, en realidad, un pasado que nunca existió en la Argentina. Nunca se dio en el país de Sarmiento una plantocracia basada en la explotación de una cuantiosa fuerza de trabajo esclavo rural. Sí, hubo esclavitud en el Río de la Plata colonial. Incluso, a diferencia de Chile, donde se había abolido la esclavitud inmediatamente después de la Independencia, en la Argentina independiente la esclavitud seguía legal en la mayor parte del país hasta la Constitución de 1853 y en Buenos Aires hasta 1861. Sin embargo, al esclavo en la Argentina se le reservaba para la servidumbre doméstica y los oficios urbanos. Para un liberal como Sarmiento que rezaba en el altar del mercado libre de trabajo de Adam Smith, la esclavitud era una institución anacrónica y anti-moderna. Entre otras cosas, funcionaba para frenar la inmigración, y por lo tanto entraba en conflicto con uno de sus ideales políticos más queridos. ${ }^{17}$

Deseo concluir este ensayo planteando un enigma. ¿Por qué este liberal, quien en la década de los cuarenta hacía la defensa del mulato como un "self-made man" potencial y quien nunca dejó de criticar la esclavitud como institución económica en el mundo moderno, perdía la oportunidad en su libro de viajes de 1849 de hablar -incluso de echar pestes como era tan a su gusto hacerlo- sobre la situación de los negros y mulatos en Cuba? Aun antes de conocer La Habana la había caracterizado, en su Facundo de 1845, como "el pueblo más rico de América, pero también el más subyugado y más degradado" (69). En otras palabras, ¿por qué guardó silencio sobre aquella parte del mundo hispano, colonia todavía de la execrada España, donde ningún blanco podía decir que el negro desaparecía como él mismo había dicho sobre la Argentina? Ningún blanco podía decirlo —si no quería pasar por loco- porque todo cuerdo estaba obligado a reconocer, por una evidencia innegable, que la presencia del negro en la isla de Cuba crecía y crecía.

\section{OBRAS CITADAS}

Andrews, George Reid. The Afro-Argentines of Buenos Aires, 1800-1900. Madison: University of Wisconsin Press, 1980.

Davis, David Brion. Slavery and Human Progress. Oxford University Press, 1984. de Tocqueville, Alexis. Vol. I de Democracy in America. Trad. Henry Reeve. Ed. Phillips Bradley. New York: Vintage, 1945.

\footnotetext{
${ }^{16}$ Un motivo constante de la última sección de Viajes es que USA representa lo más adelantado del mundo y lo que algún día alcanzará el resto de la humanidad. Ver, por ejemplo, 472, 500, 505.

${ }^{17}$ Sobre el anacronismo de la esclavitud, ver Sarmiento, "Introducción", xxv-xxvii. Para la versión sarmientina de los argumentos morales y religiosos en contra de la esclavitud, que corresponden a argumentos típicos del abolicionismo desde sus comienzos en el siglo XVIII, ver lamisma "Introducción", xix-xxi. Para el argumento de que la esclavitud pone obstáculos a la inmigración, ver Facundo, 242. Para una excelente discusión de cómo la noción del progreso funcionó para transformar las actitudes occidentales sobre la esclavitud, especialmente a partir de la abolición de la trata esclavista declarada por los ingleses en 1807, ver Davis.
} 
Endrek, Emiliano. El mestizaje en Córdoba: Siglo XVIII y principios del XIX. Córdoba: Universidad Nacional de Córdoba, 1966.

Halperín Donghi, Tulio. "Sarmiento: su lugar en la sociedad argentina post-revolucionaria". Sur 341 (julio-diciembre 1977): 121-35.

Hegel, Georg Wilhelm Friedrich. The Philosophy of History. 1832. Trad. J. Sibree. New York: Dover, 1956.

Jones, Kristine L. "Civilization and Barbarism and Sarmiento's Indian Policy". Sarmiento and His Argentina. Ed. Joseph T. Criscenti. Boulder: Lynne Rienner Publishers, 1993. 35-43.

"Indian-Creole Negotiations in the Southern Frontier". Revolution and Restoration:

The Rearrangement of Power in Argentina, 1776-1860. Ed. Mark D. Szuchman y Jonathan C. Brown. Lincoln: University of Nebraska Press, 1994. 103-23.

Kelly, Kevin. "Rosas and the Restoration of Order through Populism". Revolution and Restoration The Rearrangement of Power in Argentina, 1776-1860. Ed. Mark D. Szuchman y Jonathan C. Brown. Lincoln: University of Nebraska Press, 1994. 20839.

Klein, Herbert S. African Slavery in Latin America and The Caribbean. New York: Oxford University Press, 1986.

Knight, Franklin W. Slave Society in Cuba During the Nineteenth Century. Madison: University of Wisconsin Press, 1970.

Lynch, John. Argentine Dictator: Juan Manuel de Rosas, 1829-1852. Oxford: Clarendon, 1981.

Mander, Jerry. "The Dark Side of Globalization: What the Media Are Missing". The Nation 15/22 (July 1996): 9-14.

Rockland, Michael Aaron. "Sarmiento's Views on the United States". Sarmiento and His Argentina. Ed. Joseph T. Criscenti. Boulder: Lynne Rienner Publishers, 1993. 4550.

Sarmiento, Domingo Faustino. Aldao y El Chacho. 1845, 1868. Buenos Aires: Editorial Tor, 1938.

"Base de la democracia. Elogio de Lincoln". 14 febrero 1866. Vol. XXIX de Obras. Buenos Aires: Mariano Moreno, 1899. 231-39.

Conflicto y armonía de las razas en América. Vol. I: 1883; Vol. II: póstumo. Vols. XXXVII y XXXVIII de Obras completas. Buenos Aires: Editorial Luz del Día, 1953. "Continúa el examen del artículo Romanticismo". El Mercurio 28 julio 1842 . Vol. I de Obras. Santiago de Chile: Imprenta Gutenberg, 1887. 302-8.

"Educación de los negros libertos". s.f. Vol. XXX de Obras. Buenos Aires: Luz del Día, 1952. 231-40.

Facundo, o Civilización y barbarie. Caracas: Biblioteca Ayacucho, 1977.

"Introducción a la vida de Abran Lincoln". Vida de Abran Lincoln. Nueva York:

D. Appleton, 1866. xi-xlviii.

“"Investigaciones sobre el sistema colonial de los españoles' por J. V. Lastarria”. El

Progreso 27 setiembre 1844. Vol. II de Obras completas. Santiago de Chile: Imprenta Gutenberg, 1885. 215-22.

"Los minstrels, arte dramático popular americano". El Nacional 12 julio 1869. Vol. XXIX de Obras. Buenos Aires: Mariano Moreno, 1899. 301-4. 
“La mujery la civilización”. El Mercurio 22, 23, 24 agosto 1841. Vol. XII de Obras. Buenos Aires: Mariano Moreno, 1896. 195-213.

"El mulato, drama de Alejandro Dumas". El Mercurio 15 julio 1842. Vol. I de Obras. Santiago de Chile: Imprenta Gutenberg, 1887. 279-82. Recuerdos de provincia. 1850. Barcelona: Ramón Sopena, 1967. Viajes. 1849. Buenos Aires: Editorial de Belgrano, 1981.

Skidmore, Thomas E. "Racial Ideas and Social Policy in Brazil, 1870-1840". The Idea of Race in Latin America, 1870-1940. Ed. Richard Graham. Austin: University of Texas Press, 1990. 7-36.

Vargas Llosa, Mario. "Questions of Conquest: What Columbus Wrought, and What He Did Not”. Harper's Magazine (diciembre 1990): 45-53. 
